\title{
Estimated prognosis of ecological toxicity of new imidazolium ionic liquids with phosphorus-containing anion
}

\author{
N. Gogoleva ${ }^{1,2 *}$, O. Bernachuk ${ }^{3}$, A. Bernachuk ${ }^{3}$ \\ ${ }^{1}$ A.E. Favorsky Irkutsk Institute of Chemistry SB RAS, Irkutsk, Russia \\ ${ }^{2}$ Irkutsk Scientific Centrum SB RAS, Irkutsk, Russia \\ ${ }^{3}$ Irkutsk State University, Irkutsk, Russia \\ *e-mail: googo@mail.ru
}

Key words: ionic liquids, imidazolium hypophosphites, ecotoxicity, Quantitative structure-activity relationships (QSARs)

Motivation and Aim: Ionic liquids are increasingly used in a wide variety of fields of science and technology. In the Irkutsk Institute of Chemistry. A.E. Favorski SB RAS developed and successfully develops approaches to the directed synthesis of new functional organophosphorus compounds directly from elemental phosphorus (the Trofimov-Gusarova reaction) (see, for example, [1]), including the use of the phenomenon of defect formation in the solid structure of this chemical element [2-4]. Recently, the previously known hypophosphites 1-H-, 1-alkyl- and 1-vinyl-3H-imidazolium, which are ionic liquids at room temperature, have been obtained. The aim of the work was the calculated prediction of the environmental toxicity of these new phosphorus-containing ionic liquids.

Methods and Algorithms: Calculations were carried out using the "quantitative structure-activity relationships (QSARs)" model to evaluate the physico-chemical and environmental-toxicological properties of new ionic liquids.

Results: The model shows a group contribution method that considers three main groups of descriptors in the ionic liquid structure: the anion, the cation and the substitutions (carbon chains linked to the cation). Based on these descriptors, their contribution to the ecotoxicity of the ionic liquid has been evaluated by means of a multilinear regression model.

Conclusion: The results obtained using the QSARs model for assessing the environmental toxicity of new ionic liquids are completely correlated with the literature data for such compounds [5].

\section{References}

1. Abakumov G.A. et al. (2018) Perspective growth-points and challenges of element-organic chemistry. Russ. Chem. Rev. 87(5):393-507.

2. Gusarova N.K. et al. Reaction of vinylpyridines with active modifications of elemental phosphorus in KOH/DMSO (2001) Chem. Heterocycl. Comp. 37(5):576-580.

3. Sukhov B.G. et al. (2003) Radiation defect formation processes as a method for activation of red phosphorus in the Trofimov-Gusarova reaction. Arkivoc. 13:196-204.

4. Trofimov B.A. et al. (2006) Directed synthesis of phosphines and their derivatives based on elemental phosphorus: design of novel ligands for metal-complex catalists. Catalysis Industry. 4:18-23.

5. Luis P. et al. (2010) Quantitative structure-activity relationships (QSARs) to estimate ionic liquids ecotoxicity EC50 (Vibrio fischeri). J. Mol. Liquids. 152:28-33. 